\title{
Research Paper: Ultrasound-Guided Placement of Permanent Hemodialysis Catheters Compared to the Traditional Blind Method
}

\author{
Arash Mohammadi Tofigh ${ }^{1}$ (D), Farbod Emami Yeganeh² (D), Elham Mirzaian ${ }^{3}$ (D), Behzad Nemati Honar ${ }^{1 *}$ (iD)
}

1. Department of General Surgery, School of Medicine, Imam Hossein Hospital, Shahid Beheshti University of Medical Sciences, Tehran, Iran

2. Department of General Surgery, School of Medicine, Imam Hossein Medical Center, Shahid Beheshti University of Medical Sciences, Tehran, Iran.

3. Department of Pathology, School of Medicine, Tehran University of Medical Sciences, Tehran, Iran.

\begin{tabular}{l|l}
$\begin{array}{l}\text { Use our devic to san } \\
\text { and read thearticle online }\end{array}$ \\
manent Hemodialysis Catheters Compared to the Traditional Blind Method. International Journal of Medical Toxicology and \\
Forensic Medicine. 2019; 9(4):213-220. https://doi.org/10.32598/ijmttm.v9i4.25389
\end{tabular}

\section{(i) (5)}

Article info:

Received: 02 Mar 2019

First Revision: 25 Mar 2019

Accepted: 23 Jul 2019

Published: 01 Oct 2019

\section{Keywords:}

Dialysis, Catheters, Anatomic landmarks, Ultrasonography, Doppler

\section{A B STRACT}

Background: Central venous hemodialysis catheters are one of the most common vascular accesses for patients suffering from end-stage kidney disease. The application of ultrasoundguided placement of permanent hemodialysis catheters is highly recommended by the new guidelines because of its advantages over the traditional blind methods.

Methods: In this prospective study, the samples comprised patients $(\mathrm{N}=80)$ referred to a vascular surgery clinic by their nephrologist or elective vascular surgery consultations and asking for the placement of permanent hemodialysis catheters in Imam Hossain Hospital (A referral hospital complex center in, Tehran, Iran) during 2014 to 2015. The patients were randomly divided into two groups $(n=40)$; one of the groups underwent the placement of catheters with the guidance of Doppler ultrasound and the other with the traditional blind method. Then, the data were analyzed by SPSS V. 21 software.

Results: There was statistically significant fewer attempts for successful placement $(Z=$ $-2.725, \mathrm{P}=0.006$ ), less insertion failure (Chi-Square $=9.600, \mathrm{P}=0.002$ ), fewer failures with the first attempt (Chi-square $=9.600, \mathrm{P}=0.002)$, and less total placement time $(\mathrm{t}=1.379, \mathrm{df}=69.549$, $\mathrm{P}=0.172)$ in the sonography-guided group compared to the blind method. Furthermore, there was no significant difference in venous cannulation time and complications such as pneumothorax, neck hematoma, and carotid puncture between the two groups. Moreover, the patients had significantly greater satisfaction with the sonography-guided method $(Z=-4.306$, $\mathrm{P}=0.0001$ ).

Conclusion: According to this study, it might be beneficial to use ultrasound as the first method of permanent hemodialysis catheters insertion and patients would be more satisfied with this approach as well. In addition, learning the blind method is recommended to the practitioners because of the possibility of lacking ultrasound in an emergency.

* Corresponding Author:

Behzad Nemati Honar, MD.

Address: Department of General Surgery, School of Medicine, Imam Hossein Hospital, Shahid Beheshti University of Medical Sciences, Tehran, Iran. Tel: +98 (21) 77585851

E-mail: behzadnematihonar@yahoo.com 


\section{Introduction}

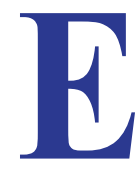

nd-Stage Kidney Disease (ESKD) is one of the most important health burdens worldwide. The total number of patients with ESKD was 350000 until 2010 based on 7\% to $9 \%$ incidents per year. Most of these patients need hemodialysis, and $70 \%$ to $85 \%$ of them prefer using intrahospital hemodialysis methods $[1,2]$. Central venous catheterization is one of the common procedures in new medicine, and it is a method for getting short or long-term venous access for hemodialysis or other diagnostic and therapeutic procedures [3, 4].

Although the best and gold-standard venous access method for hemodialysis is arteriovenous fistulas, the number of patients, who prefer using temporary or permanent hemodialysis catheters, is increased $[2,5]$. One of the most common devices is cuffed or permanent hemodialysis catheters (Permicaths), which is increasingly used for this purpose and its applying is the main aim of this study $[1,6]$.

We indicated the insertion of percutaneous dialysis catheters in the following situations [2]: 1. Patients who are waiting for arteriovenous fistula surgery or its maturation; 2 . When the arteriovenous fistula is contraindicated (such as in end-stage heart failure); 3. When life expectancy is less than a year; Those who are waiting for kidney transplantation in the immediate future.

Although the placement of these catheters is an invasive procedure, it has low but definite mortality and morbidity. Some leading causes of mortality and morbidity in this procedure include natural vascular variations, short and thick neck in obese patients, and no enough experience in catheter insertion [2]. The value of reported complications and morbidities caused by the inexperienced practitioner is $5 \%$ to $10 \%, 2 \%$ to $15 \%, 10 \%$ to $15 \%$, and even $35 \%$ [7-10].

Practitioners can place central venous cannulation devices in 3 ways [2]:

Cut down surgical method, which required an experienced person; this method is recommended because of low complication risks during insertion.

Percutaneous insertion method (Seldinger technique), which is done based on anatomic landmarks (so-called blind method) and it is the preferred method for the people who are not working in the surgical field.
Percutaneous insertion method under the guidance of sonography. Although there are various sites for central venous punctures, the right internal jugular vein is the recommended site for this procedure [2].

Nowadays, sonography is increasingly used for the placement of central venous catheters, and it is facilitated by portable bedside ultrasound devices [2].

Using landmarks for internal jugular vein puncture may have instant and significant complications. The most important and common complications are carotid artery puncture, neck hematoma, pneumothorax, and hemothorax; however, the rare complications are brain infarction because of the arterial punctures and damages to the brachial plexus, satellite ganglion, or phrenic nerve [4]. The puncture needle and its inappropriate entrance can cause all of the complications mentioned above as well [4].

All new guidelines suggest using ultrasound as the guide for placing central venous catheters (such as dialysis catheters), owing to fewer complications and fewer try and more success rates while applying color Doppler ultrasound as a guide compared to the blind method [1]. It is worth mentioning that the portable color Doppler ultrasound devices are novel in developing countries hospitals such as in this hospital (Imam Hossain Hospital, a referral hospital complex, Tehran, Iran).

\section{Objectives and goals}

The aim of this study was to compare the sonographyguided placement of permanent hemodialysis catheters (cuffed ones) with the traditional landmark method (blind method) to investigate whether the Doppler-guided placement of permanent dialysis catheters (which is considered the novel in our country) has fewer complications and more success rates or not. Should this method become the routine and the first catheter placement method? Which method is more satisfactory by the patients? Thus, we can sketch useful guidelines for our center or other health centers in our country or even other developing countries.

\section{Materials and Methods}

In this prospective randomized case-control clinical trial, we evaluated all the patients with ESKD $(\mathrm{N}=80)$ referred to the vascular surgery clinics or by intrahospital consultations asking for the placement of permanent hemodialysis catheters during 2014 to 2015 . All of the patients in this study had the experience of hemodialysis catheter insertion and the patients, who had the hemodi- 
alysis catheter for the first time, were excluded from the study in order to prevent any bias in the measurement of patients' satisfaction.

The patients were randomized by the lottery. The two groups included sonography-guided group $(n=40)$, as the case group, and the blind method group $(n=40)$, as the control group. The sonography guidance was done by Sonoscape S6 Ultrasound unit with the linear probe, in 2D neck color Doppler mode. A trained person, who was not in the insertion team, explained the purposes and advantages of the study, or even the possible complications of each insertion method and privacy considerations to the patients. All the patients signed informed consent. For the ethical considerations, patients who did not have consent to be a part of the study were excluded. As the other exclusion criterion for bias reduction, the patients who preferred to undergo a certain procedure were excluded.

We prepared the variables in a checklist, and trained people filled them and collected data in the operation rooms during the insertion. All the procedures were performed in a sterile setup and under local anesthesia. The physician ordered a Chest X-Ray (CXR) after the procedure following a post-op visit in the ward. The data were analyzed by SPSS V. 21 software. In the data analysis, we tried to stratify patients in sex, age, and neck shape to reduce more bias.

Evaluated variables and their definitions are as follow: The Number of Attempts: the number of attempts for each procedure until diagnosing that is impossible to insert the catheter by the surgeon or successful and complete placement of the catheter.

Insertion Failure: insertion failure was defined as the inability to introduce the catheter properly and completely in the central venous system despite using an appropriate technique, for any reason, from the beginning to the end of the procedure; for example, being unable to puncture the vein after multiple unsuccessful tries by the practitioner leading to the stoppage of the procedure, inability to introduce the guidewire or catheter because of any obstacle and stenosis in the path or no backflow after catheter insertion.

Failure with the first attempt: Unable to puncture the vein or any patient's related obstacles during the procedure leading to a failure to insert the catheter successfully and completely by the practitioner.
Venous cannulation time: The total time measured from the skin penetration to the successful venous puncture leading to successful catheter insertion.

Total procedure time: The time measured from the skin penetration to the complete and successful catheter entrance into the central vein.

The number of arterial punctures: The number of arterial punctures diagnosed by a pulsatile blood ejaculation through the puncture needle during the procedure.

Pneumothorax: The air accumulated between two layers of pleura diagnosed by the post-op CXR.

Hemothorax: The blood accumulated between two layers of pleura was visualized in the post-op CXR.

Neck Hematoma: Any neck hematoma observed in the post-op visit in the ward.

Patients Satisfaction: it is defined as patients' satisfaction with the overall procedure based on the patients' ease during the procedure. We designed a questionnaire, for this purpose in this study, consisting of 3 ordinal qualitative answers for 1 question (How do you rate the overall procedure based on your ease during the procedure?): Poor (considered as low satisfaction); Fair (considered as intermediate satisfaction); Excellent (considered as high satisfaction). The questionnaire was delivered to the patients by a person who was not in the insertion team.

\section{Results}

This study was carried out on 80 patients that were randomly divided into two groups (40 patients per group). The comparisons of the variable between the two groups are explained separately in detail.

\section{Descriptive statistics}

Tables 1, 2, and 3 present the descriptive results of this study.

According to Table 1, there were 3 insertion failures in the sonography-guided group (7.5\%) versus 10 in the landmark group (25\%). Additionally, there were 4 failures $(10 \%)$ with the first attempt in the sonographyguided group and $16(40 \%)$ in the landmark group. No pneumothorax or hemothorax was observed in both groups. We noted $3(7.5 \%)$ neck hematomas in the sonography-guided group in comparison to $6(15 \%)$ in the landmark group. 
Table 1. Variable frequencies

\begin{tabular}{|c|c|c|c|c|}
\hline \multirow{3}{*}{$\begin{array}{c}\text { Variable } \\
\text { Insertion failure }\end{array}$} & \multicolumn{4}{|c|}{ Frequency/Relative Frequency (\%) } \\
\hline & \multicolumn{2}{|c|}{ Sonography-Guided } & \multicolumn{2}{|c|}{ Landmark } \\
\hline & 3 & 7.5 & 10 & 25 \\
\hline Failure with the first attempt & 4 & 10 & 16 & 40 \\
\hline Pneumothorax & 0 & 0 & 1 & 2.5 \\
\hline Hemothorax & 0 & 0 & 0 & 0 \\
\hline Neck Hematoma & 3 & 7.5 & 6 & 15 \\
\hline
\end{tabular}

Table 2. The frequency rate of patients' satisfaction

\begin{tabular}{ccccc}
\hline \multirow{2}{*}{ Variable } & \multicolumn{2}{c}{ Frequency/Relative Frequency (\%) } \\
\cline { 2 - 5 } & & Sonography-Guided & \multicolumn{2}{c}{ Landmark } \\
\hline Low & 3 & 7.5 & 15 & 37.5 \\
Intermediate & 21 & 52.5 & 23 & 57.5 \\
High & 16 & 40 & 2 & 5 \\
\hline
\end{tabular}

Three patients $(7.5 \%)$ had low satisfaction from the sonography-guided group versus $15(37.5 \%)$ in the landmark group. Furthermore, 21 patients $(52.5 \%)$ had intermediate satisfaction from the sonography-guided method compared to 23 patients (57\%) satisfied with the landmark method.

According to Table 3, the Mean \pm SD number of attempts was $1.18 \pm 0.501$ in the sonography-guided group versus $1.75 \pm 1.104$ in the landmark group. The Mean \pm SD venous cannulation time was $0: 00: 34 \pm 0: 00: 35$ in the sonography-guided group compared to 0:00:48 $\pm 0: 00: 51$ in the landmark group. The total procedure time Mean \pm SD was 0:03:07 $\pm 0: 00: 44$ in the sonography-guided group and it was 0:04:03 $\pm 0: 01: 04$ in the landmark group. The
Mean \pm SD arterial puncture number was $1.15 \pm 0.533$ times in the sonography-guided group versus $0.20 \pm 0.405$ in the landmark group.

\section{Inferential statistics}

The Number of attempts

The Mean \pm SD number of attempts in all of the patients in both groups was $1.46 \pm 0.899$. Additionally, the minimum number of attempts was 1 and the maximum ones were 4 . The average rating of attempts was 35.13 in the sonography-guided group versus 45.88 in the landmark group. According to the Mann-Whitney U Test, there was a statistically significant difference in the mean rank

Table 3. The Mean $\pm S D$ of variables

\begin{tabular}{ccc}
\hline Variable & \multicolumn{1}{c}{ Mean \pm SD } & Landmark \\
\cline { 2 - 3 } Attempt numbers & Sonography-Guided & $1.75 \pm 1.104$ \\
\hline Venous cannulation time & $1.18 \pm 0.501$ & $0: 00: 48 \pm 0: 00: 51$ \\
Total procedure time & $0: 00: 34 \pm 0: 00: 35$ & $0: 04: 03 \pm 0: 01: 04$ \\
Arterial puncture & $0: 03: 07 \pm 0: 00: 44$ & $0.20 \pm 0.405$ \\
\hline
\end{tabular}


between two groups $(\mathrm{Z}=-2.725, \mathrm{P}=0.006)$. The number of attempts was fewer in the sonography-guided group.

\section{Insertion failure}

Insertion failure frequency was significantly decreased in the sonography-guided group compared to the landmark group by the Chi-square testing (Chi-square $=4.501$, $\mathrm{P}=0.034)$.

\section{Insertion failure in the first try}

According to the Chi-square test, failure in the first try frequency was significantly decreased in the sonography-guided group compared to the landmark one (Chisquare $=9.600, \mathrm{P}=0.002$ ).

\section{Venous cannulation time}

There was no significant difference in venous cannulation time between the two groups by using the Levante`s independent $\mathrm{t}$ test. $(\mathrm{t}=-1.379, \mathrm{P}=0.172)$.

\section{Total procedure time}

According to the Levante's independent $t$ test, the total procedure time was significantly lower in the sonography-guided group $(\mathrm{t}=-1.379, \mathrm{df}=69.549, \mathrm{P}=0.172)$.

\section{Pneumothorax}

We used the Chi-square test to compare the frequency of pneumothorax between two groups, but there was no significant difference between them (Chi-square $=1.013$, $\mathrm{P}=0.314$ ).

\section{Neck Hematoma}

According to the Chi-square test, there was no significant difference in neck hematoma frequency between two groups (Chi-square $=1.127, \mathrm{P}=0.288$ ).

\section{Carotid puncture}

The minimum carotid puncture frequency was zero, and the maximum one was 3 in all the patients. The carotid puncture means rank was 38.6 in the sonographyguided group, while it was 42.40 in the landmark one. Based on the Mann-Whitney U test, there was no significant difference between the two groups.

\section{Patient satisfaction}

The mean satisfaction score was 2 for all the patients, indicating that the patients had overall intermediate sat- isfaction from the procedure. The mean rank of the patients' satisfaction was 50.58 in the sonography-guided group, while it was 30.43 in the landmark group. According to the Mann-Whitney $U$ test, people are significantly more satisfied with the sonography-guided method compared to the landmark method $(\mathrm{Z}=-4.306, \mathrm{P}=0.0001)$.

\section{Discussion}

As we mentioned earlier, according to the results of the present study, the number of attempts was significantly decreased in the sonography-guided group, and it is compatible with Prabhu et al.'s study in 2010 [11]. Therefore, we recommend using sonography as a guide, especially when the patients' physical or mental condition mandates insertion with fewer attempts.

The insertion failure was significantly reduced in the sonography-guided group, and it is compatible with the findings of Denys et al. in 1993 [12]. Thus, we recommend sonography as a guide notably when the insertion failure will cause a major burden for the patient and in an emergency as well.

The failure in the sonography-guided group in addition to direct visualization and insertor experience might be because of previous catheter insertions that lead to anatomical variations and complications, such as anatomical venous angulation or stenosis that could make the insertion impossible and end up with insertion under venography visualization or surgery. As Marteen W. Taal et al. reported, central vein stenosis happens in some patients, who had previous tunneled hemodialysis catheters that might warrant fluoroscopy and venography for a safe and thorough insertion [13].

According to these findings, failure with the first try was significantly decreased in the sonography-guided group. This finding is compatible with the studies of Rabindranath et al. and Denys et al. [1, 12]. We do emphasize using ultrasound as a guide in an emergency when the patient does not have enough physical and mental readiness.

As explained in the previous paragraphs, there was no significant difference in venous cannulation time between two groups, and it is not in line with the findings of Nadig et al. [14]. This result might be because of the practitioners' expertise in blind method insertion that led to a fast venous cannulation time.

The total procedure time was statistically lower in the sonography-guided method, and this result is compat- 
ible with the findings of Nadig et al. [14]. Since there was no statistical difference in venous cannulation time between the two groups, the lower total procedure time might be because of the fewer number of attempts in the sonography-guided group.

Based on our findings, there were no differences in complications such as pneumothorax, hemothorax, neck hematoma, or carotid puncture between the two groups. This finding is in contrast with the studies of Rabindranath et al. and Denys et al. [1, 12]. Nadig et al. found no difference in pneumothorax between the two groups as well; but in their study, neck hematoma was significantly decreased in the sonography-guided group. The previous applying of this method by the practitioners might lead to our results.

The patients' satisfaction rate was obviously higher from the sonography-guided method $(Z=-4.306$, $\mathrm{P}=0.0001$ ). Thus, we suggest ultrasound as the first approach to have more patients with higher satisfaction.

Furthermore, according to the results, we can conclude that using color Doppler ultrasound as a guide for permanent hemodialysis catheter insertion will lead to quicker catheter insertion and a reduction in the number of attempts compared to the blind method, which is one of the main concerns in an emergency.

\section{Conclusion}

We have conceded that color Doppler ultrasound might not be available in every emergency room and emergency in general, especially in the disadvantaged areas; hence, we do suggest learning and teaching the blind method for every practitioner.

This study could not determine the best insertion method. Choosing a proper insertion approach might be varied for different patients because of the differences in the practitioners' expertise in each method, equipment, time limit, and patients' moral and physiological situation. The practitioners must select the proper method by evaluating the above-mentioned factors.

Each insertion method could be selected as the first insertion method, depending on the practitioners' choice. According to the recommendations of international organizations, such as NICE2002 and Renal Association 2007 for using sonography-guided insertion as the first method, and by considering its advantages such as more patients' satisfaction, it appears that sonography-guided method should be chosen as the first insertion method [7, 15].
The results of this study could be generalized to other central venous catheter insertion methods. We recommend more evaluation for the other central venous catheters too.

We propose further studies to reveal the causes, which make the practitioners stop the procedure and consider it failed and clarify the exact anatomical obstacles by venography after the insertion failure.

Moreover, we recommend further investigations based on the patients' age, sex, and body mass index to revealing the effects of individual characteristics on the study's outcomes. Further evaluation warrants evaluating the history of catheter insertion as a variable too.

Furthermore, we recommend standardized questionnaire-based studies, focusing just on patient's satisfaction in order to make a thorough questionnaire about these two approaches.

In this study, insertion failure was just based on practitioners' conclusion; we recommend other investigations with the aim of showing the variables that may affect practitioners to stop the procedure such as, practitioners' tiredness or low patients' compliance. Therefore, we could acquire a more precise definition of insertion failure.

\section{Ethical Considerations}

\section{Compliance with ethical guidelines}

The research protocol approved by the Ethics Committee of Shahid Beheshti University of Medical Sciences, Tehran, Iran. Informed consent form was obtained from all study subjects.

\section{Funding}

This research was conducted by personal funds and we received no specific grant from any funding agency in the public, commercial, or not-for-profit sectors.

\section{Author's contributions}

Designing the study, approving final version of the manuscript and accountable for all aspects of the work: All authors; Participating in acquisition of data: Farbod Emami Yeganeh, Elham Mirzaian; Analyzing data: Arash Mohammadi Tofigh, Farbod Emami Yeganeh; Managing data: Behzad Nemati Hhonar, Arash Mohammadi Tofigh, Farbod Emami Yeganeh; Writing the first 
draft and other critically revise: Farbod Emami Yeganeh, Elham Mirzaian.

\section{Conflict of interest}

The authors declared no conflict of interes

\section{References}

[1] Mactier R, Hoenich N, Breen C. Renal association clinical practice guideline on haemodialysis. Nephron Clinical Practice. 2011; 118(Suppl. 1):c241-c86. [DOI:10.1159/000328072] [PMID]

[2] Rabindranath KS, Kumar E, Shail R, Vaux E. Use of real-time ultrasound guidance for the placement of hemodialysis catheters: A systematic review and meta-analysis of randomized controlled trials. American Journal of Kidney Diseases. 2011; 58(6):964-70. [DOI:10.1002/14651858.CD005279.pub4] [PMID]

[3] Serafimidis K, Sakorafas GH, Konstantoudakis G, Petropoulou K, Giannopoulos GP, Danias N, et al. Ultrasound-guided catheterization of the internal jugular vein in oncologic patients; comparison with the classical anatomic landmark technique: A prospective study. International Journal of Surgery. 2009; 7(6):526-8. [DOI:10.1016/j.ijsu.2009.08.011] [PMID]

[4] Kumar A, Chuan A. Ultrasound guided vascular access: Efficacy and safety. Best Practice \& Research Clinical Anaesthesiology. 2009; 23(3):299-311. [DOI:10.1016/j.bpa.2009.02.006]

[5] Pisoni RL, Gillespie BW, Dickinson DM, Chen K, Kutner $\mathrm{MH}$, Wolfe RA. The Dialysis Outcomes and Practice Patterns Study (DOPPS): Design, data elements, and methodology. The American Journal of Kidney Diseases. 2004; 44(5 Suppl. 2):7-15. [DOI:10.1053/j.ajkd.2004.08.005] [PMID]

[6] Ethier J, Mendelssohn DC, Elder SJ, Hasegawa T, Akizawa T, Akiba T, et al. Vascular access use and outcomes: An international perspective from the dialysis outcomes and practice patterns study. Nephrology Dialysis Transplantation. 2008; 23(10):3219-26. [DOI:10.1093/ndt/gfn261] [PMID] [PMCID]

[7] Natoinal Institute for Health and Care Excellence. Guidance on the use of ultrasound locating devices for placing central venous catheters. London: National Institute for Clinical Excellence; 2002.

[8] Bailey PL, Glance LG, Eaton MP, Parshall B, McIntosh S. A survey of the use of ultrasound during central venous catheterization. Anesthesia \& Analgesia. 2007; 104(3):491-7. [DOI:10.1213/01.ane.0000255289.78333.c2] [PMID]

[9] Leung J, Duffy M, Finckh A. Real-time ultrasonographically-guided internal jugular vein catheterization in the emergency department increases success rates and reduces complications: A randomized, prospective study. Annals of Emergency Medicine. 2006; 48(5):540-7. [DOI:10.1016/j.annemergmed.2006.01.011] [PMID]

[10] Shojania KG, Duncan BW, McDonald KM, Wachter RM, Markowitz AJ. Making health care safer: A critical analysis of patient safety practices. Evidence Report/Technology Assessment (Summary). 2001; (43):i-x 1-668. [PMID] [PMCID]

[11] Prabhu MV, Juneja D, Gopal PB, Sathyanarayanan M, Subhramanyam S, Gandhe S. Ultrasound-guided femoral dialysis access placement: A single-center randomized trial. Clinical Journal of the American Society of Nephrology. 2010; 5(2):235-9. [DOI:10.2215/CJN.04920709] [PMID] [PMCID]

[12] Denys B, Uretsky B, Reddy P. Ultrasound-assisted cannulation of the internal jugular vein. A prospective comparison to the external landmark-guided technique. Circulation. 1993; 87(5):1557-62. [DOI:10.1161/01.CIR.87.5.1557] [PMID]

[13] Taal MW, Chesterton LJ, McIntyre CW. Venography at insertion of tunnelled internal jugular vein dialysis catheters reveals significant occult stenosis. Nephrology Dialysis Transplantation. 2004; 19(6):1542-5. [DOI:10.1093/ndt/gfh216] [PMID]

[14] Nadig C, Leidig M, Schmiedeke T, Höffken B. The use of ultrasound for the placement of dialysis catheters. Nephrology Dialysis Transplantation. 1998; 13(4):978-81. [DOI:10.1093/ ndt/13.4.978] [PMID]

[15] Ashby D, Borman N, Burton J, Corbett R, Davenport A, Farrington $\mathrm{K}$, et al. Renal association clinical practice guideline on haemodialysis. BMC Nephrology. 2019; 20:379. [DOI:10.1186/s12882-019-1527-3] [PMID] [PMCID] 
This Page Intentionally Left Blank 